\title{
AULAS DE ENLACE: ESTUDIO DE LA IMPLANTACIÓN DE UN PROGRAMA EDUCATIVO EXPERIMENTAL PARA RECIÉN LLEGADOS EN LA COMUNIDAD DE MADRID
}

\author{
Ma DOLORES INGLÉS LÓPEZ \\ Centro Superior de Idiomas. Universidad de Alicante \\ mdist6@hotmail.com
}

\begin{abstract}
Resumen
Este artículo expone las conclusiones del estudio llevado a cabo sobre la implantación del programa Escuelas de Bienvenida en la Comunidad de Madrid (CAM), centrándose en las «Aulas de Enlace» como parte integrante fundamental del mismo. El estudio analiza las experiencias y percepciones de los participantes en el programa, identifica los factores que influyen en dichas percepciones, analiza el impacto del programa en el aprendizaje de los alumnos, y documenta sus intenciones de continuar en el sistema educativo hacia la educación no obligatoria. Asimismo, intenta explicar la conexión entre la política educativa oficial y las experiencias de los participantes en el programa. El estudio enfatiza la importancia de dar voz a todos los participantes en programas educativos innovadores, a la vez que pretende promover un diálogo que resulte en políticas y prácticas educativas más informadas y capaces de atender de manera eficaz las necesidades reales de los alumnos inmigrados.
\end{abstract}

PALABRAS CLAVE: evaluación de programas educativos, enseñanza y aprendizaje de segundas lenguas, educación de minorías inmigradas, alumnado extranjero recién llegado.

\begin{abstract}
This article investigates the implementation of the Escuelas de Bienvenida program («Welcome Schools»), in the Autonomous Community of Madrid (CAM). Specifically, it focuses on the «Aulas de Enlace», one integral part of the program. This research study investigates the participants' everyday experiences and the perceptions and meanings attached to those experiences while in the program. Additionally, it identifies the factors that affect participant perceptions, analyzes the impact of the program on student learning and documents their intentions to continue to post-compulsory education. The study also attempts to uncover the match between the official policy and the participants' lived experiences and perceptions of the program. The study underscores the importance of giving voice to the constituents of educational innovations. In doing so, I hope to promote conversation that will lead to more thoughtful and informed policy making and practice.
\end{abstract}

KEY WORDS: language program evaluation, newcomer students, second language teaching and learning, immigrant minority education.

\section{Introducción}

Como consecuencia de procesos migratorios determinados por cambios políticos y socioeconómicos, la población inmigrante ha crecido de manera considerable en España en las últimas dos décadas. En este periodo Madrid ha jugado un papel decisivo como receptor de flujos migratorios (Lora-Tamayo, 2003), ya que constituye, junto con Barcelona, uno de los grandes mercados laborales del país.

La Tabla 1.1 muestra la evolución de la población extranjera en la CAM desde 2001. 
Tabla 1.1. Cifras de población inmigrante en España y la CAM

\begin{tabular}{|l|c|c|c|c|}
\hline & \multicolumn{2}{|c|}{ Padrón de Habitantes } & \multicolumn{2}{c|}{ Censo de Población } \\
\hline & 2001 & 2008 & 2001 & 2008 (estimado) \\
\hline ESPAÑA & 1.370 .657 & 5.220 .577 & 1.548 .941 & 5.220 .600 \\
\hline CAM & 305.656 & 991.259 & 362.617 & $882.293(2007)$ \\
\hline
\end{tabular}

La mayor presencia de trabajadores inmigrantes ha resultado también en una mayor presencia de sus hijos en nuestras escuelas, principalmente como resultado del proceso de reunificación familiar y/o el incremento del número de nacimientos en familias inmigradas. Como consecuencia, las aulas españolas han experimentado un rápido y continuo proceso de diversificación respecto a los países de procedencia del alumnado y al número de lenguas presentes en el contexto escolar. Si bien la escuela es el lugar donde primero se enfrentan los problemas de inclusión/exclusión (Soriano Ayala, 2000), así como el lugar ideal para sentar las bases de una verdadera sociedad multicultural, en la realidad se han realizado pocos esfuerzos reales de adaptación para enfrentarse a un aula cada vez más heterogénea.

El programa Escuelas de Bienvenida surge como idea en noviembre de 2002 y se implanta finalmente en enero de 2003 con la finalidad de dar respuesta a la creciente diversidad en las aulas de la CAM. Aunque en un primer momento se presenta como un proyecto educativo innovador, las autoridades lo definen como «una nueva medida de educación compensatoria» (www.madrid.org), donde el término «compensar» nos trae a la memoria programas basados en teorías del déficit que el nuevo programa estaría tratando de superar.

\subsection{Objetivos del estudio}

El principal objetivo del estudio fue explorar la implantación del programa Escuelas de Bienvenida a través de un análisis profundo de uno de sus componentes principales, esto es, las denominadas «Aulas de Enlace». Las «Aulas» fueron originalmente ideadas para dos tipos de alumnado extranjero, hispanohablantes con desfases curriculares de mayor o menor grado, y alumnos con desconocimiento del castellano. Un tercer grupo lo constituirían los alumnos no hispanohablantes con desfase curricular. Este trabajo de investigación no se ocupó del alumnado hispanohablante, ya que mi interés principal se centra en el aprendizaje de segundas lenguas. De igual modo, tampoco se tomaron en consideración los datos académicos procedentes de la evaluación directa del proceso de aprendizaje lingüístico de los alumnos, que será objeto de futuras investigaciones. En muchas ocasiones estos datos objetivos y medibles simplemente no existían o eran de exclusivo uso interno del profesorado.

Las preguntas de investigación que el estudio trató de responder son las siguientes:

1. ¿Cuáles son las experiencias en el proceso de aprendizaje de los participantes en el programa?

2. ¿Cuáles son las percepciones y los significados ligados a dichas experiencias?

3. ¿Cuál es el impacto del programa en la intención de continuar los estudios más allá de la educación obligatoria?

4. ¿Qué factores son los que más influyen en las percepciones de alumnos y profesores en el programa? 
5. ¿De qué modo coincide la política oficial sobre el programa con las experiencias y las percepciones de alumnos y profesores?

\subsection{Justificación del estudio}

A pesar de que el programa Escuelas de Bienvenida se presentó en el año 2003 como un «programa experimental», la política oficial carece de criterios claros y sistemáticos de evaluación y seguimiento, algo absolutamente necesario en cualquier tipo de «experimento» que se lleve a cabo. Este estudio pretende en cierta medida llenar ese vacío, lo que lo convierte en especialmente relevante y oportuno.

La evaluación de programas específicos para alumnos inmigrantes es un área de gran interés en países con altos porcentajes de población extranjera (Valladares, 2005; Short \& Boyson, 2004; Plaut \& Sharkey, 2003; Short, 2002; Thomas \& Collier, 2002, 1997; Walqui, 2000; Hakuta, K. et al., 2000). Sin embargo, y de acuerdo con Short (2002), no es un área suficientemente investigada, a pesar de que dicha investigación es fundamental para delimitar las características de un buen programa educativo para este grupo de alumnado en continuo crecimiento.

Este trabajo de investigación pretende demostrar la importancia de la evaluación rigurosa de los programas educativos, más allá de la comprobación de su buen funcionamiento administrativo. Para ello se realizó una descripción detallada de la implantación del programa Escuelas de Bienvenida, es decir, una descripción de cómo es realmente el programa en funcionamiento. En este proceso de descripción el estudio recoge las voces de alumnos y profesores, además de las voces de los dirigentes y administradores de la política educativa, lo que lo convierte en uno de los pocos estudios que lo hace en el contexto español. Para Nieto (1994: 395) «One way to begin the process of changing school policies and practices is to listen to students' views about them.» A pesar de esto, y de acuerdo con la autora, las investigaciones que toman en cuenta las voces de los estudiantes son escasas y relativamente recientes.

\subsection{Breve descripción del programa}

El programa Escuelas de Bienvenida se implantó el 20 de enero de 2003 en un total de 115 centros públicos y privados concertados de educación primaria y secundaria de la CAM. En julio de 2003 se dictaron las denominadas «Instrucciones», un documento de doce páginas y trece directivas que regula el funcionamiento del programa, y donde se especifica el marco legal que justifica la creación de programas educativos específicos para población extranjera. De este modo se establece que el principal objetivo de las «Aulas» es «posibilitar la atención específica al alumnado extranjero con desconocimiento del idioma español o con un grave desfase curricular que se incorpora a lo largo del curso escolar, apoyando la adquisición de competencias lingüísticas y comunicativas, y desarrollando el proceso de enseñanza y aprendizaje mediante las oportunas adaptaciones curriculares.» (www.madrid. org). Las «Instrucciones» han sido revisadas cada año para introducir leves modificaciones e incluir documentación oficial de uso administrativo (consentimiento de padres, informes de seguimiento y evaluación, etc.) 
La escolarización del alumnado en las «Aulas de Enlace» se lleva a cabo a través de las «Comisiones de Escolarización» de cada distrito de la CAM, y el programa es voluntario, siendo la aceptación por escrito del padre, madre o tutor del alumno requisito imprescindible para participar en el mismo. El programa atiende a alumnado con edades comprendidas entre los 6 y los 16 años (hasta los 18 años en casos excepcionales), en clases de un máximo de 12 alumnos, y durante un periodo de permanencia de entre seis meses y un año. Los alumnos del «Aula» reciben inicialmente clases intensivas de español, a la vez que se intenta facilitar su pronta incorporación a su correspondiente aula ordinaria en clases como tecnología, educación física y/o música.

Las «Instrucciones» no establecen criterios de evaluación inicial, más allá de ser extranjero y/o no hablante nativo de español, o final, mediante la cual valorar la adecuación de la incorporación de un alumno a su aula de referencia u ordinaria. Respecto al profesorado encargado del programa, el trabajo de clase es compartido por dos profesores, uno de los cuales es el tutor o coordinador del «Aula». Normalmente uno de los profesores se encarga de la enseñanza de castellano, mientras que el otro se hace cargo de la enseñanza de asignaturas como matemáticas, sociales o conocimiento del medio. No existe un currículum específico para el «Aula de Enlace», por lo que son los profesores los que determinan los contenidos a impartir y los métodos usados para hacerlo según su propio criterio. Esta circunstancia resulta con frecuencia en un mayor énfasis en destrezas como la expresión oral y la comprensión lectora. Cada profesor imparte un máximo de quince horas lectivas semanales y rara vez coinciden ambos en el aula durante una misma clase.

La promoción de actividades extraescolares (excursiones, actividades culturales o eventos especiales) es uno de los pilares sobre los que se asienta el programa Escuelas de Bienvenida, ya que persigue la interacción con los alumnos del aula ordinaria fuera del contexto escolar formal. En la realidad, son pocos los alumnos del aula ordinaria que participan en actividades promovidas por el «Aula de Enlace», aunque cada alumno del «Aula» debe participar en las que organiza su aula de referencia u ordinaria.

\section{Metodología}

El trabajo de investigación que aquí se presenta se inscribe dentro del paradigma cualitativo. Aunque hace uso de un instrumento de recogida de datos tradicionalmente cuantitativo como es la encuesta o cuestionario, y reduce y analiza los datos usando estadística descriptiva, asume los principales postulados del paradigma cualitativo. Tal y como los formula Creswell (1994: 4-10), estos postulados son los siguientes: «First, reality is subjective and multiple as seen by participants in the study ... value-laden and biased ... context-bound, and accurate and reliable through verification. Second, the researcher interacts with that being researched. Third, the nature of the problem is an important factor ... and the research problem needs to be explored because little information exists on the topic».

Dentro del paradigma cualitativo, el estudio fue diseñado como un estudio de caso de orientación fenomenológica en el que se explora una única entidad, es decir, las «Aulas de Enlace». Creswell (1994) considera la fenomenología como una de las grandes tradiciones cualitativas. Según Van Manen (1990), el objetivo de la fenomenología es alcanzar una mejor comprensión de la naturaleza o el significado de nuestras experiencias cotidianas, lo que para Creswell (1998) es totalmente aconsejable si el objetivo es explorar un fenómeno sobre el 
que poco se sabe aún. Para Walqui (2000: 93), uno de los aspectos más positivos de hacer investigación cualitativa es hecho de que «there may be many sources and different viewpoints represented», por lo que, según la autora, este tipo de investigación debería fomentarse.

\subsection{Papel del investigador}

A lo largo de este estudio yo fui el principal instrumento de recogida y análisis de datos, y llevé a cabo su posterior interpretación. Así pues, contacté con los centros y los profesores de «Aulas de Enlace» de toda la CAM, distribuí y recogí los cuestionarios, entrevisté a los participantes (con ayuda de intérpretes cuando fue necesario), realicé observación participante y no participante en las clases, recogí toda la información relevante a este estudio en diferentes cuadernos de notas (cuadernos de observación de clases, de aplicación de la metodología, y de incidencias durante el trabajo de campo), y examiné los documentos disponibles sobre el programa.

Mi deseo de dar voz a los alumnos y forma a sus experiencias en el programa viene en parte determinada por mi propia experiencia como inmigrante en EEUU. Aunque este hecho podría considerarse un riesgo para la objetividad del estudio, al mismo tiempo me hizo tomar una conciencia clara de mi papel como investigadora y de la necesidad de comprender y juzgar las experiencias de todos los participantes con imparcialidad. Asimismo, mi propia experiencia como profesora de español trajo consigo el riesgo de realizar juicios de valor respecto a los métodos de enseñanza de la lengua, por lo que en todo momento tuve extremo cuidado por describir las interacciones tal y como sucedieron en clase de manera objetiva evitando en todo momento valoraciones personales.

\subsection{Descripción de los participantes}

El método de selección de los participantes en el estudio fue el muestreo intencionado, es decir, la selección basada en una razón o característica específicas. Para Merrian (1988) esta técnica de muestreo es común en los estudios de caso, donde el objetivo es la investigación detallada de un grupo y no la generalización de los resultados. El requisito para participar en el estudio fue únicamente formar parte del programa Escuelas de Bienvenida como alumno, profesor o administrador. En el caso de los alumnos se establecieron cuatro criterios: (1) ser recién llegado (menos de 9 meses de residencia en España); (2) estar matriculado en un «Aula de Enlace» en el curso 2004-2005; (3) tener entre 12 y 18 años de edad, y (4) ser hablante nativo de rumano, árabe/bereber o chino.

Un total de 116 alumnos participaron en el estudio, de los cuales 34 fueron rumanos, 37 marroquíes y 45 chinos. La elección de estas tres nacionalidades en concreto se basó en la creencia de que son representativas de la diversidad de la escuela secundaria pública en la CAM. En esta investigación las tres nacionalidades representan lados opuestos del éxito en el aprendizaje del castellano. Así, mientras los alumnos rumanos alcanzan un nivel de lengua oral aceptable al poco tiempo de su llegada, los alumnos chinos mantienen una lucha abierta con la lengua durante la mayor parte de su educación secundaria. Respecto a los alumnos marroquíes, muchos alcanzan un buen nivel de competencia oral, aunque presentan severas dificultades en la escritura y en diversas áreas curriculares. La Tabla 2.1 muestra el perfil de los alumnos participantes en la investigación. 
Tabla 2.1 Perfil del alumnado participante

\begin{tabular}{|c|c|c|c|c|}
\hline & $\begin{array}{c}\text { Rumanos } \\
\mathrm{N}=34\end{array}$ & $\begin{array}{c}\text { Chinos } \\
\mathrm{N}=45\end{array}$ & $\begin{array}{c}\text { Marroquíes } \\
\qquad \mathrm{N}=37\end{array}$ & $\begin{array}{l}\text { TOTAL } \\
\mathrm{N}=116\end{array}$ \\
\hline \multicolumn{5}{|l|}{ Sexo } \\
\hline Hombre & $47 \%$ & $40 \%$ & $49 \%$ & $46 \%$ \\
\hline Mujer & $53 \%$ & $60 \%$ & $51 \%$ & $54 \%$ \\
\hline \multicolumn{5}{|l|}{ Edad } \\
\hline 12-14 años & $59 \%$ & $31 \%$ & $29.7 \%$ & $40 \%$ \\
\hline 15-17 años & $41 \%$ & $67 \%$ & $67.5 \%$ & $58.5 \%$ \\
\hline$>17$ años & $0 \%$ & $2 \%$ & $2.7 \%$ & $1.5 \%$ \\
\hline \multicolumn{5}{|l|}{ Año académico } \\
\hline $1^{\circ} \mathrm{ESO}$ & $26.5 \%$ & $17.8 \%$ & $13.5 \%$ & $19.5 \%$ \\
\hline $2^{\circ} \mathrm{ESO}$ & $35.3 \%$ & $24.5 \%$ & $16.2 \%$ & $25.5 \%$ \\
\hline $3^{\circ} \mathrm{ESO}$ & $8.8 \%$ & $26.7 \%$ & $51.3 \%$ & $29 \%$ \\
\hline $4^{\circ} \mathrm{ESO}$ & $29 \%$ & $31 \%$ & $16.2 \%$ & $25.5 \%$ \\
\hline \multicolumn{5}{|c|}{ Última clase en país de origen } \\
\hline No escolarizado & $0 \%$ & $0 \%$ & $16.2 \%$ & $5.4 \%$ \\
\hline Educación primaria & $17.6 \%$ & $9 \%$ & $30 \%$ & $19 \%$ \\
\hline $1^{\circ} \mathrm{ESO}$ & $30 \%$ & $24.5 \%$ & $10.8 \%$ & $22 \%$ \\
\hline $2^{\circ} \mathrm{ESO}$ & $12 \%$ & $29 \%$ & $13.5 \%$ & $18 \%$ \\
\hline $3^{\circ} \mathrm{ESO}$ & $20 \%$ & $37.8 \%$ & $2.7 \%$ & $20 \%$ \\
\hline $4^{\circ} \mathrm{ESO}$ & $15 \%$ & $0 \%$ & $10.8 \%$ & $8.6 \%$ \\
\hline \multicolumn{5}{|l|}{ Tiempo en España } \\
\hline Hasta 7 meses & $29.5 \%$ & $26.8 \%$ & $40.7 \%$ & $32.3 \%$ \\
\hline 8 meses o más & $70.5 \%$ & $73.2 \%$ & $59.4 \%$ & $67.7 \%$ \\
\hline \multicolumn{5}{|l|}{ Tiempo en su IES } \\
\hline Hasta 6 meses & $47 \%$ & $49 \%$ & $49 \%$ & $48.3 \%$ \\
\hline 6 meses o más & $53 \%$ & $51 \%$ & $51 \%$ & $51.7 \%$ \\
\hline \multicolumn{5}{|l|}{ Tiempo en su «Aula» } \\
\hline Hasta 6 meses & $94 \%$ & $49 \%$ & $78 \%$ & $73.7 \%$ \\
\hline 6 meses o más & $6 \%$ & $51 \%$ & $22 \%$ & $26.3 \%$ \\
\hline
\end{tabular}


Un total de 36 profesores participaron en el estudio, de los cuales casi un $70 \%$ fueron mujeres con edades comprendidas entre los 41 y los 50 años. Un 75\% de ellos admitió tener más de 20 años de experiencia en la enseñanza, y tan sólo una media de 0-4 años en la enseñanza de español a extranjeros (el 70\% de la muestra). Todos los profesores habían recibido un curso de 25 horas organizado por la consejería de educación de la CAM, formación que la mayoría consideró insuficiente. Un 61\% de los profesores llevaban más de dos años en el programa, mientras que un 39\% había estado en el programa al menos un año, con la intención de continuar en la totalidad de los casos.

En cuanto a los administradores del programa, tres directores de IES y dos inspectores de educación participaron en el estudio. Como representantes de la Consejería de Educación de la CAM fueron entrevistados la directora del departamento de Promoción Educativa, Dña Ma Antonia Casanova y el entonces Consejero de Educación, D. Luis Peral Guerra.

\subsection{Descripción de los centros (IES)}

El estudio se llevó a cabo en 23 centros públicos de educación secundaria de toda la CAM, cuatro de los cuales fueron seleccionados como estudios de caso (ver descripción en Tabla 2.2).

Tabla 2.2. Descripción detallada de los cuatro IES seleccionados como estudio de caso

\begin{tabular}{|c|c|c|c|c|}
\hline & IES1 & IES2 & IES3 & IES4 \\
\hline Observación & $\begin{array}{c}15 \text { nov-15 dic. } \\
2004\end{array}$ & $\begin{array}{c}17 \text { enero- } 1 \text { febr. } \\
2005\end{array}$ & $\begin{array}{c}\text { 4-9 febr. y } \\
15-30 \text { marzo } 2005\end{array}$ & $\begin{array}{l}31 \text { mar-7 abril } \\
2005\end{array}$ \\
\hline $\begin{array}{l}N^{0} \text { alumnos y } \\
\text { edades }\end{array}$ & $\begin{array}{c}6 \\
\text { (edades: 12-14) }\end{array}$ & $\begin{array}{c}11 \\
\text { (edades: } 12-18)\end{array}$ & $\begin{array}{c}9 \\
\text { (edades: 12-16) }\end{array}$ & $\begin{array}{c}10 \\
\text { (edades: 14-17) }\end{array}$ \\
\hline $\begin{array}{l}\text { Composición del } \\
\text { alumnado }\end{array}$ & $\begin{array}{l}3 \text { Rumanos } \\
1 \text { Chinos } \\
1 \text { Iraní } \\
1 \text { Dominicano }\end{array}$ & $\begin{array}{l}2 \text { Rumanos } \\
2 \text { Búlgaros } \\
3 \text { Chinos } \\
2 \text { Marroquíes } \\
1 \text { Marfileño } \\
1 \text { Ucraniano }\end{array}$ & $\begin{array}{l}7 \text { Chinos } \\
2 \text { Búlgaros }\end{array}$ & $\begin{array}{l}2 \text { Rumanos } \\
1 \text { Marroquíes } \\
6 \text { Chinos } \\
1 \text { Ucranianos }\end{array}$ \\
\hline Edad profesores & $\begin{array}{l}\text { P1: 40-45 años } \\
\text { P2: 40-45 años }\end{array}$ & $\begin{array}{l}\text { P3: 55-60 años } \\
\text { P4: 45-50 años }\end{array}$ & $\begin{array}{l}\text { P5: 40-45 años } \\
\text { P6: } 30-35 \text { años }\end{array}$ & $\begin{array}{l}\text { P7: 40-45 años } \\
\text { P8: 50-55 años }\end{array}$ \\
\hline $\begin{array}{l}\text { Especialidad del } \\
\text { profesorado }\end{array}$ & $\begin{array}{l}\text { P1: Ciencias } \\
\text { Naturales } \\
\text { P2: Matemáticas }\end{array}$ & $\begin{array}{l}\text { P3: Inglés } \\
\text { P4: Matemáticas }\end{array}$ & $\begin{array}{l}\text { P5: Inglés } \\
\text { P6: Lengua y lite- } \\
\text { ratura española }\end{array}$ & $\begin{array}{l}\text { P7: Inglés } \\
\text { P8: Ciencias } \\
\text { sociales }\end{array}$ \\
\hline $\begin{array}{l}\text { Horario de } \\
\text { profesores }\end{array}$ & Independiente & $\begin{array}{l}\text { Compartido ( } 2 \\
\text { horas) }\end{array}$ & Independiente & $\begin{array}{l}\text { Compartido ( } 2 \\
\text { horas) }\end{array}$ \\
\hline $\begin{array}{l}\text { Lenguas } \\
\text { que habla el } \\
\text { profesorado }\end{array}$ & $\begin{array}{l}\text { Inglés y } \\
\text { castellano }\end{array}$ & $\begin{array}{l}\text { Inglés, francés y } \\
\text { castellano }\end{array}$ & Inglés y castellano & $\begin{array}{l}\text { Inglés, francés, } \\
\text { portugués y } \\
\text { castellano }\end{array}$ \\
\hline
\end{tabular}




\begin{tabular}{|c|c|c|c|c|}
\hline & IES1 & IES2 & IES3 & IES4 \\
\hline $\begin{array}{l}\text { Uso de otras len- } \\
\text { guas en clase }\end{array}$ & NO & $\begin{array}{c}\text { SÍ } \\
\text { (Inglés y } \\
\text { francés) }\end{array}$ & NO & $\begin{array}{l}\text { Sí (palabras suel- } \\
\text { tas y expresiones } \\
\text { en L1 de los } \\
\text { alumnos ) }\end{array}$ \\
\hline Localización IES & $\begin{array}{l}\text { Clase media y } \\
\text { media alta }\end{array}$ & Clase media & $\begin{array}{l}\text { Clase media baja } \\
\text { o trabajadora }\end{array}$ & $\begin{array}{l}\text { Clase media baja } \\
\text { o trabajadora }\end{array}$ \\
\hline $\begin{array}{l}\text { Localización del } \\
\text { «Aula» en el } \\
\text { centro }\end{array}$ & $\begin{array}{l}\text { Integrada } \\
\text { con aulas de } \\
\text { compensatoria }\end{array}$ & Aislada & $\begin{array}{l}\text { Integrada con au- } \\
\text { las ordinarias }\end{array}$ & $\begin{array}{l}\text { Integrada con au- } \\
\text { las ordinarias }\end{array}$ \\
\hline $\begin{array}{l}\text { Incorporación } \\
\text { tardía alumnos }\end{array}$ & $\begin{array}{l}\text { SÍ (1 alumno } \\
\text { dominicano) }\end{array}$ & $\begin{array}{l}\text { SÍ ( } 2 \text { alumnas } \\
\text { chinas) }\end{array}$ & $\begin{array}{l}\text { SÍ ( } 2 \text { alumnas } \\
\text { chinas y una } \\
\text { marfilense) }\end{array}$ & $\begin{array}{l}\text { SÍ (1 alumna } \\
\text { ucrania) }\end{array}$ \\
\hline $\begin{array}{l}\text { Enseñanza } \\
\text { de lengua / } \\
\text { contenido }\end{array}$ & $\begin{array}{l}\text { P1: lengua } \\
\text { P2: contenido }\end{array}$ & $\begin{array}{l}\text { P3: lengua }+ \\
\text { contenido } \\
\text { P4: lengua }+ \\
\text { contenido }\end{array}$ & $\begin{array}{l}\text { P5: lengua + } \\
\text { contenido } \\
\text { P6: lengua }\end{array}$ & $\begin{array}{l}\text { P7: lengua + } \\
\text { contenido } \\
\text { P8:contenido }\end{array}$ \\
\hline
\end{tabular}

$\mathrm{P}=$ profesor/a

Aunque se pretendió en todo momento contar con la participación de centros privados concertados con alumnado inmigrante, esta opción tuvo que ser desestimada finalmente bien por la falta de respuesta a mis peticiones, bien por los muchos obstáculos que se pusieron a mi entrada en los centros.

La selección de los IES participantes se realizó de acuerdo con dos criterios: (1) el número de alumnos matriculados en el «Aula de Enlace» del centro en ese momento, y en particular aquellos de origen chino, rumano y marroquí; y (2) el grado de accesibilidad y la disposición de los directores, profesores y alumnos a participar en el estudio.

\subsection{Cuestiones éticas}

Tanto el trabajo de campo en los cuatro centros seleccionados como estudios de caso, como la administración de cuestionarios y entrevistas en el resto de centros educativos se llevó a cabo tras obtener el permiso de entrada de los directores de los centros mediante carta firmada y sellada. Asimismo, se preparó un consentimiento informado en cada una de las lenguas de los alumnos participantes en el estudio dirigido a los padres o tutores. Este consentimiento se adjuntó al cuestionario y se envió a casa con cada uno de los alumnos. Una vez firmado el consentimiento por el padre o tutor, el alumno completó el cuestionario y lo devolvió al profesor de su «Aula de Enlace», que a su vez se lo hizo llegar a la investigadora.

Todos los instrumentos de recogida de datos en este estudio protegen el anonimato de los participantes, y en ningún momento se desvelan nombres o datos que pudieran revelar su identidad. 


\subsection{Técnicas de recogida de datos}

Los datos para esta investigación se recogieron de diversas maneras y desde diferentes puntos de vista con el fin de describir el programa desde múltiples perspectivas, incluida la de la investigadora. Para ello desarrollé cuestionarios y entrevistas semiestructuradas específicas para alumnos y profesores y entrevistas semiestructuradas para los administradores participantes en el programa.

\subsubsection{Cuestionarios}

Dado que los cuestionarios tenían como objetivo recoger opiniones y percepciones sobre un programa muy específico, tuve que desarrollar un modelo de cuestionario ad hoc para este estudio y traducirlo a las tres lenguas de los alumnos participantes, es decir, chino, rumano y árabe, para lo que contraté los servicios de traductores profesionales.

Los cuestionarios de alumnos y profesores tienen estructuras similares y ambos incluyen 83 preguntas abiertas y cerradas breves. Las preguntas cerradas están divididas en seis escalas con los siguientes contenidos:

(1) información personal: edad, sexo, país de origen, fecha de llegada, planes en España, tipo de centro, etc.;

(2)_lengua materna: nivel de conocimiento, patrones de uso, grado de mantenimiento, preferencias de uso en la escuela y actitudes hacia su L1;

(3) segunda lengua (español): nivel de conocimiento, motivación para su aprendizaje, valores asignados a la L2, uso, y actitudes hacia la lengua y sus hablantes;

(4) tu «Aula de Enlace»: razones para estar en el programa, expectativas de aprendizaje, objetivos a conseguir, opiniones sobre la escuela, los profesores, los materiales usados en clase, tipo de programa, sentimientos respecto a su incorporación al aula ordinaria;

(5) planes de futuro: planes después de la educación obligatoria, objetivos personales y el uso futuro del español;

(6) padres: profesión de los padres (en España y en su país de origen), nivel de español, ayuda que reciben de ellos en las tareas escolares, opiniones de los padres sobre la educación de sus hijos en general y sobre el programa y el aprendizaje de la lengua en particular, planes de futuro para sus hijos.

Las preguntas cerradas consisten en escalas de Likert con cuatro opciones, preguntas de verdadero o falso, preguntas a las que asignar un valor numérico y preguntas de opción múltiple. Las preguntas abiertas consisten en preguntas específicas para ser contestadas en una sola línea. Por ejemplo: «Las tres palabras que describen a los chinos son: " o «Las tres cosas que menos te gustan de tu 'Aula de Enlace'

son: El tiempo estimado para completar los cuestionarios es de

entre 30 y 45 minutos.

Los cuestionarios del alumno y el profesor tuvieron una fase de pilotaje con cinco participantes entre los alumnos y tres entre los profesores. Tras esta fase se realizaron los cambios oportunos en determinados ítems antes de llevar a cabo su administración a gran escala. 


\subsubsection{Entrevistas}

Se realizaron entrevistas a cada uno de los participantes en el programa, o a una muestra representativa de ellos en el caso de alumnos y profesores. El objetivo de estas entrevistas fue volver a explorar algunos de los temas tratados en el cuestionario con el fin de reafirmar o rechazar los datos obtenidos en él. Por esta razón su estructura es muy similar a la de las encuestas, esta vez con cinco escalas (pues se omite la información personal) y una batería de preguntas abiertas por cada escala.

Las entrevistas se realizaron en la lengua nativa de los alumnos y para ello requerí los servicios de intérpretes profesionales (a veces miembros de ONGs que se ofrecieron voluntarios para colaborar en mi estudio) o de hablantes, entre los propios estudiantes extranjeros, con un alto nivel de conocimiento del castellano.

Tanto los alumnos como los profesores fueron seleccionados para realizar entrevistas de entre aquellos que respondieron afirmativamente a la pregunta final del cuestionario, esto es, «¿te importaría que te llamáramos para una entrevista de seguimiento? De este modo, ocho alumnos y cinco profesores fueron entrevistados y grabados con su consentimiento. Los datos grabados fueron transcritos para su posterior análisis de contenido.

\subsubsection{Observación}

Según King et al. (1987: 85), «Most audiences consider the observations of people [...] highly credible sources of information about program implementation», y esto es así porque la observación pone al investigador lo más cerca posible del funcionamiento del programa.

La observación, tanto participante como no participante, para este estudio se llevó a cabo en los cuatro IES seleccionados desde mediados de noviembre de 2004 hasta finales de abril de 2005 durante un total de 164 horas. En este periodo de tiempo se registró todo lo ocurrido en las aulas a través de notas tomadas en diferentes cuadernos con diferentes propósitos. Además de esto, las frecuentes visitas a los 23 centros participantes me permitieron mantener conversaciones informales con educadores, directores y secretarios/as, las cuales me ayudaron a tener una idea más clara del contexto en el que se implanta el programa.

\subsubsection{Notas de campo}

Se tomaron notas de todo lo ocurrido en el programa durante todas las fases de este estudio con el fin de suplementar la información obtenida a través de las observaciones, cuestionarios y entrevistas. Se recogieron tres tipos de notas: (1) notas descriptivas, esto es, datos sobre el comportamiento de los participantes y el contexto; (2) notas metodológicas, para documentar el funcionamiento y/o las incidencias de los métodos de recogida de datos usados en el estudio, y (3) notas analíticas, que incluyeron mis propias ideas y percepciones sobre el programa y la organización escolar.

\subsubsection{Análisis de documentación}

El análisis de documentos ha sido una constante en este estudio desde antes incluso de empezar el trabajo de campo y la recogida de datos. Los primeros documentos analizados 
correspondieron a la legislación educativa en la que se apoya la implantación del programa Escuelas de Bienvenida, esto es, LOGSE, LOCE, Plan Regional de Compensación Educativa de la CAM, «Instrucciones» del programa, además de algunos de los Proyectos Curriculares de Centro de los IES donde se llevó a cabo la observación. Por el contrario, no se tuvo acceso a las programaciones de los profesores o a sus planes de clase, así como a las notas o exámenes. Sin embrago, sí tuve acceso a distintos tipos de materiales didácticos usados en clase y ejemplos de materiales producidos por los alumnos en el «Aula de Enlace»

Otro tipo de documentación analizada fueron datos estadísticos disponibles en las páginas web del Ministerio de Educación (www.mec.es) y la consejería de educación de la CAM (www.madrid.org), tales como cifras de estudiantes en el programa por año de implantación y distribución geográfica.

\subsection{Verificación de datos}

Con el fin de evitar el riesgo de introducir cualquier tipo de prejuicio o idea preconcebida en la fase de recogida, análisis y/o interpretación de los datos, y así aumentar la solidez de los resultados del estudio, se establecieron unos criterios claros de fiabilidad y se emplearon una serie de técnicas para aumentar la validez de los resultados (Guba y Lincoln, 1989). De este modo, el estudio da cuenta de la «credibilidad» con el uso del «member checking» y técnicas de triangulación. Además de esto, la credibilidad, también denominada «validez interna» en estudios cualitativos, se refuerza por la presencia continuada y persistente, es decir, la inmersión en el campo y el establecimiento de buenas relaciones con los participantes en el estudio con el fin de entender sus percepciones. Respecto a la validez externa del estudio, esta se consiguió a través de una clara y completa descripción del programa, lo que se conoce como «thick description», esto es «a literal description of the entity being evaluated, the circumstances under which it is used, the characteristics of the people involved in it, the nature of the community in which it is located, and the like» (Guba y Lincoln, 1989: 119).

\subsection{Técnicas de análisis de datos}

\subsubsection{Estadística descriptiva}

Para Dörnyei (2003: 114), «descriptive statistics are used to summarize sets of numerical data in order to conserve time and space.» Por tanto, este tipo de análisis estadístico no permite sacar conclusiones más allá de la muestra o generalizar los resultados de un estudio, por lo que Dörnyei (2003) sugiere comenzar cada oración de nuestro análisis descriptivo con la frase «In my sample ...» (en mi/esta muestra). Puesto que este estudio no pretende generalizar sus resultados más allá de la muestra, sólo se usó este tipo de análisis estadístico.

Dos tipos de datos se obtuvieron del cuestionario, esto es, nominales o categóricos (los participantes se clasifican con palabras) y ordinales o intervalos (aquellos en los que los participantes se clasifican de acuerdo con un rango de intervalos numéricos). Los procedimientos estadísticos usados para reducir estos datos fueron los porcentajes y, ocasionalmente, la media aritmética, definida como el punto de equilibrio en una distribución. 


\subsubsection{Análisis de contenido}

Este tipo de análisis se utiliza en investigación cualitativa para describir, analizar y resumir las tendencias observadas en documentos escritos, incluidas las entrevistas y las notas de campo. Para Patton (2002: 453) «content analysis is used to refer to any qualitative data reduction and sense-making effort that takes a volume of qualitative material and attempts to identify core consistencies and meanings»».

En este estudio se analizó el contenido de las respuestas a las preguntas abiertas en los cuestionarios de alumnos y profesores, las transcripciones de las entrevistas a todos los participantes, las respuestas de los profesores en la fase de member checking, diversos documentos y notas de campo.

\subsubsection{Member checking.}

Para Jonhstone (2000: 65), «Member checking involves asking the people you are studying whether your analysis of their behavior is right.» Así pues, el objetivo principal de esta fase de análisis fue asegurar que la realidad construida a través de las observaciones, cuestionarios, entrevistas y análisis de documentos concordaba con la de los participantes en el programa. Se trata, pues, de una técnica muy efectiva para reducir posibles prejuicios en la interpretación de los resultados.

El proceso de member checking comenzó con mis primeras visitas a los centros, en los que mantuve frecuentes conversaciones informales con alumnos y profesores, y concluyó con member checks individuales a dos profesores del programa en IES no seleccionados como estudio de caso (ambas mujeres).

Esta parte consistió en entrevistas individuales de entre 45 minutos y 1 hora de duración que fueron grabadas y posteriormente transcritas. Las profesoras fueron informadas del objetivo y el procedimiento de la entrevista y de los métodos de obtención de datos en el estudio, así como de las respuestas de los profesores participantes y de los primeros resultados del análisis. En todo momento se les solicitó su feedback y se respondió a las preguntas surgidas. Este diálogo fue recogido en notas informales.

\subsection{Temporización del estudio}

Tabla 2.3. Fechas de las fases del estudio

\begin{tabular}{|c|c|c|c|c|}
\hline \multicolumn{2}{|c|}{ Curso 2003-2004 } & \multicolumn{3}{|c|}{ Curso 2004-2005 } \\
\hline Abril-Junio & Nov.-Dic. & Enero-Febrero & Abril-Mayo & Junio-Julio \\
\hline Documentación & $\begin{array}{c}\text { Acceso a las } \\
\text { escuelas }\end{array}$ & $\begin{array}{c}\text { Observaciones y } \\
\text { notas }\end{array}$ & $\begin{array}{c}\text { Observaciones y } \\
\text { notas }\end{array}$ & \\
\hline $\begin{array}{l}\text { Contacto con } \\
\text { escuelas }\end{array}$ & $\begin{array}{c}\text { Observaciones y } \\
\text { notas }\end{array}$ & $\begin{array}{l}\text { Encuestas alum- } \\
\text { nos y profesores }\end{array}$ & & \\
\hline \multirow[t]{2}{*}{$\begin{array}{c}\text { Contacto con } \\
\text { administradores }\end{array}$} & $\begin{array}{l}\text { Instrumentos lis- } \\
\text { tos y traducidos }\end{array}$ & & $\begin{array}{l}\text { Entrevistas alum- } \\
\text { nos y profesores }\end{array}$ & $\begin{array}{c}\text { Entrevistas } \\
\text { administradores }\end{array}$ \\
\hline & $\begin{array}{c}\text { Piloto cuestionario } \\
\text { de alumnos }\end{array}$ & & & \\
\hline
\end{tabular}




\section{Resultados}

Esta parte del estudio se divide en cinco secciones, y cada una de las cuales pretende dar respuesta a las preguntas de investigación propuestas en la introducción de este trabajo. La primera sección describe la práctica real del aula tal y como se observó en las cuatro «Aulas de Enlace» en las que se llevaron a cabo observaciones, mientras que la segunda parte trata las experiencias y percepciones lingüísticas y educativas de los participantes en el programa. La tercera sección se ocupa de los factores que más han influido en dichas experiencias y percepciones, y da respuesta a la pregunta de investigación 3. La cuarta sección investiga si, y en su caso cómo, la política educativa coincide con la práctica real y los objetivos establecidos por el programa. La última sección se ocupa de investigar el impacto del programa en las intenciones de los alumnos de continuar sus estudios más allá de la educación obligatoria.

\subsection{Práctica de aula}

Esta sección presenta de manera esquemática (ver Tabla 3.1) una descripción de la práctica de aula real en cada uno de los IES donde se realizó observación. Para esta descripción se tuvieron en cuenta los siguientes bloques: (1) los patrones de interacción en el aula; (2) el tipo de feedback correctivo; (3) la clase de input recibido; (4) el uso y tipo de materiales empleados, y (5) la planificación y los métodos de evaluación.

La calidad de la interacción en el aula es una variable importante en el aprendizaje de una segunda lengua, ya que este ocurre como resultado de las oportunidades de interactuar con otros en la L2 (véase como ejemplo Pica, 1994; Hatch, 1992; Long, 1983; Vygotsky, 1978). El análisis de la interacción se ocupó del uso de la L1, el papel del profesor, el tipo de input que recibe el alumnado y la forma de presentarlo (simplificado, mediante explicaciones, etc.), la existencia de negociación del significado, los patrones de interacción más frecuentes o el uso de comunicación no verbal.

Tabla 3.1. Resumen de la práctica de aula ( $P=$ profesor $)$

\begin{tabular}{|c|c|c|c|c|c|c|c|c|}
\hline & \multicolumn{2}{|c|}{ IES1 } & \multicolumn{2}{|c|}{ IES2 } & \multicolumn{2}{|c|}{ IES3 } & \multicolumn{2}{|c|}{ IES4 } \\
\hline & P1 & P2 & P3 & P4 & P5 & P6 & P7 & P8 \\
\hline INTERACCIÓN & P-St & - & P-St & P-St & P-St & P-St & P-St & P-St \\
\hline Uso de L1 & \multicolumn{2}{|c|}{$\begin{array}{l}\text { muy poco } \\
\text { (sólo alumnos) }\end{array}$} & \multicolumn{2}{|c|}{$\begin{array}{l}\text { muy poco } \\
\text { (sólo alumnos) }\end{array}$} & \multicolumn{2}{|c|}{$\begin{array}{l}\text { Lengua de comunicación } \\
\text { (alumnos chinos) }\end{array}$} & \multicolumn{2}{|c|}{$\begin{array}{c}\text { Muy poco } \\
\text { (alumnos y P7) }\end{array}$} \\
\hline Papel del profesor & controlador & - & Facilitator & facilitador & Facilitator & controlador & Facilitador & controlador \\
\hline $\begin{array}{l}\text { Dirigido por profesor } \\
\text { /Centrado en alumno }\end{array}$ & Profesor & - & Profesor & Profesor & Profesor & Profesor & Profesor & Profesor \\
\hline $\begin{array}{l}\text { Referential questions- } \\
\text { Display questions }\end{array}$ & $\begin{array}{l}\text { No efectiva } \\
\sqrt{ }\end{array}$ & $\begin{array}{l}- \\
-\end{array}$ & $\begin{array}{l}\sqrt{ } \\
\sqrt{ } \text { (gramática) }\end{array}$ & $\begin{array}{l}\sqrt{ } \\
\sqrt{ }\end{array}$ & $\begin{array}{l}\sqrt{ } \\
\sqrt{ }\end{array}$ & $\begin{array}{l}X \\
V \\
\end{array}$ & $\begin{array}{l}\text { V } \\
X \\
\end{array}$ & $\begin{array}{l}\sqrt{ } \\
\sqrt{ }\end{array}$ \\
\hline $\begin{array}{l}\text { Teacher talk / Input } \\
\text { modificado }\end{array}$ & \begin{tabular}{|l|}
$\sqrt{ }$ \\
$(90 \%)$ \\
\end{tabular} & $\begin{array}{l}- \\
-\end{array}$ & $\begin{array}{ll}\sqrt{ } & (50 \%) \\
\end{array}$ & & $\sqrt{ }$ & $0 \%)$ & $\begin{array}{c}\sqrt{ } \\
(50 \%)\end{array}$ & $\begin{array}{l}\text { poco } \\
(90 \%) \\
\end{array}$ \\
\hline $\begin{array}{l}\text { Modificación de la } \\
\text { interacción }\end{array}$ & Muy poca & - & $\sqrt{ }$ & $\sqrt{ }$ & Poca & Poca & $\sqrt{ }$ & Poca \\
\hline
\end{tabular}




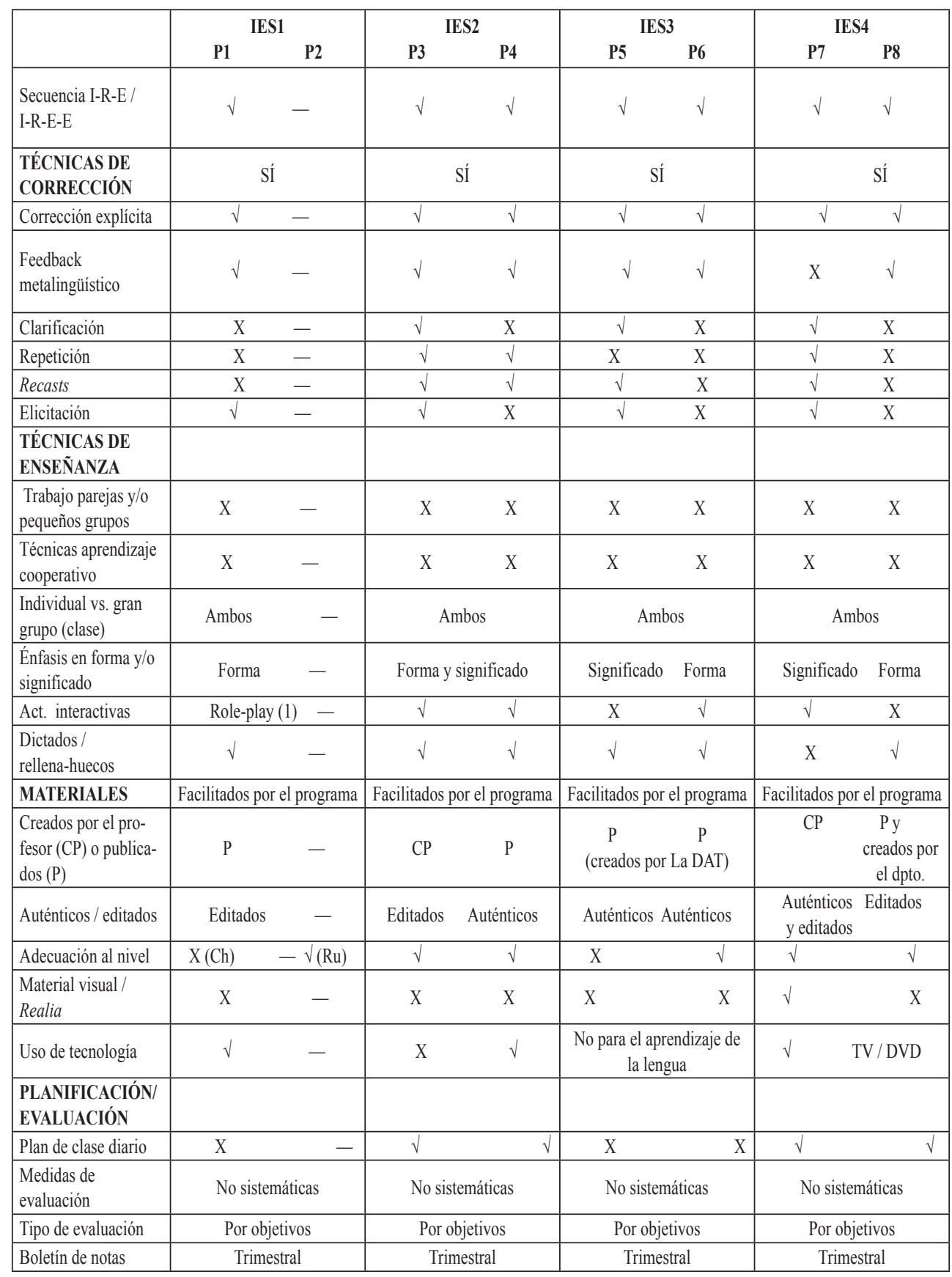




\subsection{Experiencias y percepciones de los participantes en el programa}

En los datos analizados en esta sección se encontró que las percepciones de los alumnos sobre sus experiencias en el programa son muy similares a las de sus profesores, mientras que tanto las percepciones de profesores como las de los alumnos difieren de aquellas de los administradores educativos y aquellos que elaboran la política educativa y deciden sobre su implantación. Los siguientes temas surgieron del análisis de datos:

(1) El papel de la L1 se limita a un uso privado y la escuela no se percibe como responsable de su mantenimiento. Además, las L1 de los alumnos son consideradas como el mayor obstáculo para el aprendizaje y la integración en el caso de alumnos marroquíes y chinos especialmente, por lo que no juegan papel alguno en el proceso de aprendizaje del castellano.

(2) Los datos revelaron grandes diferencias en cuanto a los niveles de conocimiento de la lengua y los procesos de adquisición en los tres grupos. Todos los grupos percibieron el aprendizaje del castellano como una prioridad, ya que de ello depende su éxito en el sistema educativo. De igual modo, todos los participantes estuvieron de acuerdo en que el aprendizaje de la L2 puede acelerarse cuando se enseña de manera intensiva. La enseñanza del contenido no se plantea como una posibilidad real durante su estancia en el «Aula», ya que habría que hacer cambios sustanciales en la escuela que requerirían gran cantidad de tiempo y esfuerzo.

(3) El grado de consecución de los objetivos del programa es percibido de diferente manera por los grupos de participantes en el estudio. Entre los alumnos, esta percepción varía dependiendo de las nacionalidades, aunque todos consideraron que el programa sí les está ayudando a aprender la lengua. Para los profesores, el programa ayuda a los alumnos chinos y marroquíes a conseguir un nivel básico de castellano, mientras que los alumnos rumanos son los que más aprovechan la experiencia, ya que son capaces de adquirir un nivel avanzado de lengua con facilidad. Para los administradores del programa y los responsables de la política educativa, el aprendizaje de L2 se lleva a cabo en seis meses («en seis meses hablan y se integran») o incluso menos para algunos alumnos, mientras que los alumnos chinos son los únicos que pueden necesitar mayor tiempo de aprendizaje intensivo.

\subsection{Impacto de programa en su intención de continuar en la educación post-obligatoria}

En general, el análisis de datos reveló que hay claras diferencias con respecto a los planes de futuro entre los tres grupos de alumnos participantes. Mientras que los alumnos rumanos se muestran muy interesados en continuar su educación más allá del nivel obligatorio, para después asistir a la universidad, los alumnos marroquíes y chinos se inclinan más por la formación profesional o por abandonar los estudios una vez concluida la educación obligatoria. Sin embargo, existen también claras diferencias entre alumnos chinos y marroquíes. Aunque algunos de los alumnos chinos de la muestra mostraron gran interés por seguir estudios postobligatorios, admiten que no es probable que esto ocurra, bien a causa del plan migratorio trazado para ellos por sus familias, o por sus dificultades con el aprendizaje del castellano. La gran mayoría de alumnos marroquíes que se mostraron interesados en la educación postobligatoria se inclinaron por la formación profesional, esto es, una formación que les dé acceso a un trabajo en el menor tiempo posible. 
Cuando se les pregunta a profesores y administradores sobre las intenciones de continuidad de sus alumnos, ambos coinciden en que la motivación de los alumnos para proseguir sus estudios está en clara relación con factores como sus aspiraciones en el país de acogida, su estatus socio-económico y su L1, que de algún modo puede facilitar o entorpecer su aprendizaje de L2. Por tanto, el programa «Aulas de Enlace» parece tener un impacto positivo en aquellos alumnos cuyo proceso migratorio incluye formación universitaria y cuya L1 es muy similar al castellano, es decir, los alumnos rumanos. Además, se observa un pesimismo generalizado respecto a lo que la escuela puede, o debe, hacer para revertir esta situación, ya que se cree que no tiene ningún control sobre ciertos factores que son los que, en definitiva, condicionan el éxito o el fracaso académico de los alumnos.

\subsection{Factores que más influyeron en las percepciones de alumnos y profesores}

El análisis de datos desveló que los factores que más influyen en las percepciones de los particpantes en el estudio son: (1) la distancia entre la L1 y el castellano; (2) las aspiraciones del alumno y su familia en España y su estatus socio-económico en el país de origen; (3) la edad de llegada a España, y (4) el tiempo de educación formal recibida en el país de origen. Estos factores han sido establecidos con claridad en la literatura académica para la misma población (Lightbown y Spada, 1999; Oxford y Shearin, 1994; Wong-Fillmore, 1991a; Long, 1990; Odlin, T, 1989; Cummins, 1984; Cummins, 1981b; Gardner y Lambert, 1972; Lenneberg, 1967), por lo que puede decirse que estos factores son algunos de los atributos esenciales para comprender a los estudiantes extranjeros inmigrantes de nuestras escuelas, considerando que pueden producir diferentes niveles de rendimiento académico.

En numerosas ocasiones, algunos de los factores considerados en esta sección son percibidos como fijos y difíciles de cambiar por parte de profesores y administradores. Esta percepción ha dado como resultado la clasificación de los alumnos de acuerdo con su capacidad para aprender L2 y para integrarse. Existe un riesgo real de que los administradores perciban estos factores como los causantes del desigual nivel de logro de los alumnos, y que consideren que la escuela tiene un papel muy limitado en el éxito del alumnado inmigrante.

\subsection{Coincidencia de percepciones y objetivos que plantea la política educativa}

Esta sección ha investigado la relación entre política educativa y práctica a través de las perspectivas de los diferentes participantes en el programa. De esta relación surgen tres ideas principales: (1) el hecho de que los legisladores y administradores del programa con poder de decisión muchas veces trabajan como meros ejecutivos, definiendo los problemas en base a la opinión pública y ofreciendo lineas de actuación muy amplias respecto a la implantación; (2) si bien el proceso de elaboración de la política educativa ha dejado de lado a los profesores, a ellos se les hace responsables de cualquier incidencia sobre el programa. Este hecho ha provocado la desconfianza de los profesores respecto a cualquier idea nueva o política que viene de las autoridades educativas de la CAM; y (3) la política oficial no parece haber tenido un impacto en la práctica educativa en el «Aula de Enlace», aunque tampoco fue concebida para ello originalmente. 


\section{Discusión}

Este apartado resume y discute las tres principales conclusiones de este estudio: (1) las contradicciones entre el diseño del programa, su implantación y la práctica real de aula y la literatura académica sobre adquisición de segundas lenguas y educación de inmigrantes; (2) la adecuación del programa Escuelas de Bienvenida, y las «Aulas de Enlace» en particular, para todos y cada uno de los estudiantes a los que va dirigido; y finalmente (3) la falta de una planificación e implantación sistemática del programa.

\subsection{Contradicciones entre diseño, implantación y práctica y la literatura académica}

El debate sobre cual es el mejor modo de enseñar a los alumnos pertenecientes a minorías inmigradas ha producido una gran cantidad de literatura académica en todo el mundo. Según Cummins (2000: 39), «a substantial research and theoretical basis for policy decisions regarding minority students' education does exist». Por tanto, parece razonable pensar que se pueden predecir con bastante grado de fiabilidad los efectos de los programas para alumnado inmigrante en muy diversos contextos socio-políticos. Además de esto, la investigación en adquisición de segundas lenguas ha propuesto múltiples teorías para explicar las maneras más efectivas para aprender una segunda lengua en contextos escolares, a la vez que ha desarrollado métodos para llevar estas teorías a la práctica de aula (Hall y Verplaetse, 2000; Larsen-Freeman, 2000; Shrum y Glisan, 2000; Lee y VanPatten, 1995).

La gran cantidad de literatura académica existente en las dos áreas mencionadas arriba puede informar la planificación, el diseño y la implantación de las «Aulas de Enlace» de varias maneras. En primer lugar, proporcionando el conocimiento previo para predecir cómo el programa puede funcionar con esta población en un determinado contexto. En segundo lugar, ayudando a evitar falsas ideas que no representan las necesidades de los alumnos y presentan enfoques simplistas para el aprendizaje de L2. Finalmente, presentando diferentes filosofías, diseños y técnicas de instrucción de programas ejemplares que abordan los retos de los alumnos inmigrantes.

Esta sección tiene por objetivo la revisión de algunas falsas ideas que guiaron la implantación del programa «Aulas de Enlace», además de discutir las implicaciones de las contradicciones entre teoría, implantación y práctica para el éxito del programa.

\subsubsection{Tiempo necesario para aprender castellano}

La política oficial de las «Aulas de Enlace» establece «acortar el periodo necesario para la integración de este alumnado al sistema educativo español» como uno de sus objetivos principales. Con esta intención en mente se reduce el periodo de estancia en el «Aula» a seis meses, en los que el alumno deberá no sólo aprender la lengua sino también los contenidos curriculares.

Cummins (1979) distinguió entre dos importantes aspectos del desarrollo de la L2: (1) BICS (basic interpersonal communication skills) y (2) CALP (cognitive academic language proficiency). BICS se refiere a la fluidez conversacional, que la mayoría de alumnos alcanza en dos años. En cuanto a CALP, esto es, el tipo de lengua que se requiere conocer en el contexto académico, los alumnos necesitan al menos cinco años para desarrollar niveles de 
CALP adecuados a su edad. Otros autores que apoyan la distinción BICS/CALP son Coelho (2004), Hakuta (2000) y Thomas y Collier $(1995,2002)$. El tiempo necesario para adquirir la L2 académica varía de un alumno a otro, y depende de una gran variedad de factores y características del alumnado, un hecho que no fue lo suficientemente evaluado por los administradores con poder de decisión antes de la implantación del programa. Durante el trabajo de campo se observó que la falta de información sobre el alumnado producía una gran frustración a los profesores, que no saben cómo atender las necesidades de sus alumnos de un modo más eficaz. Además, el límite de tiempo impuesto para la estancia en el programa no tomó en cuenta la distinción entre los dos niveles de conocimiento de la lengua (oral/comunicativo vs. académico), asumiendo que una mayor exposición a la L2 aceleraría el proceso de adquisición.

\subsubsection{El aprendizaje de la L2 como prioridad absoluta}

De acuerdo con las tres aproximaciones a la planificación lingüística de Ruiz (1984), las «Aulas de Enlace» pertenecerían a la categoría de «lengua como problema», donde el castellano debería aprenderse lo antes posible, pues representa el principal obstáculo para la integración en la vida escolar. Según Cummins (1992: 134): «when the majority culture educators look at minority children they tend to focus on what those children lack, and usually what they see is the absence of a high level proficiency in the majority language and knowledge of the majority culture.» Es quizá por esta razón por la que el programa «Aulas de Enlace» no ha integrado ningún tipo de enseñanza de contenido con la enseñanza de L2, aunque la literatura académica (véase Cenoz and Genesee, 1998; Genesee, 1994) haya mostrado que la integración de lengua y contenido es más efectiva que la enseñanza exclusiva de L2.

\subsubsection{Tipo de programa y calidad de la enseñanza}

El papel de la L1 del alumno en su educación ha sido objeto de gran cantidad de investigaciones (Cummins 1979a y b, 1983, 1992b, 2000; Lambert y Tucker, 1972; Ramírez, 1992; Swain y Lapkin, 1991b; Thomas y Collier, 1997, 2002; Tucker y d'Anglejan, 1972;). Estas investigaciones han demostrado que los alumnos aprenden mejor en su lengua materna, y que pueden pensar en un nivel más abstracto y manejar ideas más complicadas en la lengua que mejor conocen, esto es, su(s) L1(s). A pesar de que tanto profesores como administradores están convencidos de las ventajas del bilingüismo para el futuro de los alumnos en el programa, no creen que la escuela deba hacerse responsable de la enseñanza y desarrollo de su(s) lengua(s) materna(s).

\subsection{Adecuación del programa para los alumnos participantes}

El término «adecuación» se refiere aquí al grado en que las «Aulas de Enlace» consiguen sus objetivos de (1) desarrollar el nivel de español de los alumnos; (2) integrar a los alumnos al aula regular/ordinaria; y (3) reducir el tiempo de integración del alumnado. 


\subsubsection{Objetivo 1: aprendizaje de la segunda lengua}

Durante las horas de observación y a través de los datos obtenidos mediante diferentes instrumentos desarrollados especialmente para este estudio, se halló que los alumnos obtienen diferentes niveles de castellano dependiendo de factores, como la distancia entre su L1 y el castellano, sus aspiraciones, la edad de llegada o el papel de la educación en sus planes de futuro. Los diferentes niveles de logro emergieron a partir de varios instrumentos desarrollados para este estudio: (1) las percepciones de profesores y alumnos sobre el nivel de español en cuestionarios y entrevistas; (2) un instrumento de evaluación del nivel de L2 para profesores mostró que estos percibían que sólo un 50\% de la muestra de alumnos había obtenido o llegaría a obtener un nivel de L2 suficiente para poder tener éxito en el sistema educativo español; y (3) la cantidad de tiempo de permanencia en el «Aula» y el número de clases al que asiste en el aula ordinaria también son indicativos de los diferentes niveles de logro.

\subsubsection{Objetivo 2: Integración}

El término integración aquí se refiere a dos aspectos de la experiencia del alumno, es decir (1) la integración al aula ordinaria; y (2) la integración como adaptación al sistema educativo español. La integración está íntimamente conectada con el primer objetivo de aprendizaje de la lengua, ya que se consigue como consecuencia del conocimiento de L2. Por tanto, y dado que la integración al aula ordinaria no se produce hasta que se ha conseguido un cierto nivel de L2, podemos considerar que este objetivo tampoco se consigue más que para aproximadamente un $50 \%$ del alumnado.

Con respecto al segundo aspecto del objetivo de integración, también se encontraron diferencias entre los grupos de estudiantes, y el hecho de que los alumnos carecieran de oportunidades para interactuar con sus compañeros del centro parece tener una relevancia especial. Si bien las «Aulas de Enlace» se encuentran integradas en la escuela, la mayoría permanecen ajenas a la vida escolar.

\subsection{Falta de sistematización en la planificación y la implantación del programa.}

Esta sección discute la tercera idea del estudio, es decir, la idea de que la planificación, el diseño y la implantación del programa Escuelas de Bienvenida no se llevó a cabo de manera rigurosa sino que fue en gran medida fruto de la improvisación. La evidencia que apoya esta afirmación es el análisis de documentos, la observación no participante, y el análisis de datos de las entrevistas a los administradores participantes.

\subsubsection{Planificación y diseño del programa}

En su estudio sobre los programas para recién llegados en USA, Short y Boyson (2004: 51) consideran que «developing a newcomer program needs to be a thoughtful, informed and iterative process.» Para las autoras, desarrollar un programa supone, entre otras cosas, investigar sobre las diferentes opciones de implantación, llegar a una variedad de grupos que puedan beneficiarse del programa, visitar programas ya en funcionamiento, y aplicar toda 
esa información a la elaboración de un programa que cubra las necesidades y objetivos de los alumnos.

Cuando se les preguntó sobre el rationale detrás de la elección de este tipo de programa en particular («ESL-type» program), los responsables de la política educativa y la administración del programa no fueron capaces de ofrecer argumentos sólidos que justificaran su respuesta. Tampoco las «Instrucciones» proporcionan ningún tipo de marco teórico que apoye la selección de este tipo de programa sobre otros. Para los administradores del programa es obvio que el aprendizaje de la lengua es la prioridad absoluta de los alumnos recién llegados, y que su conocimiento es la base para su integración en el sistema educativo español.

\subsubsection{Implantación}

La falta de una planificación sistemática ha dado como resultado una serie de carencias que han afectado a la implantación de programa y han impactado de diversas maneras en la educación de los alumnos de minorías inmigradas. En primer lugar, cuestiones como los retos del alumnado chino con la L2, el tiempo necesario para alcanzar un nivel adecuado de castellano, o la educación de los alumnos con desfases curriculares añadidos al desconocimiento de la lengua vehicular no fueron anticipados, lo que no ha impedido que se conviertan en grandes obstáculos para los docentes. Además, estos obstáculos han ido forjando las percepciones de los profesores respecto a lo que sus alumnos saben realmente y lo que serán capaces de conseguir en el sistema educativo español.

La segunda carencia que, desde mi punto de vista, surge de la falta de planificación sistemática es la selección del profesorado. Para Short y Boyson (2004: 70) «recruiting and hiring experienced teaching staff who are trained to address the special needs of new immigrant students is a guarantee for program success.» En el caso de las «Aulas de Enlace» no hubo tiempo para la selección de profesorado que cumpliera con esas características. De este modo, el primer año académico de implantación (2002-2003) los profesores eran interinos casi en su totalidad. El segundo año y siguientes los profesores fueron seleccionados entre aquellos que mostraron interés por enseñar en el programa, y de acuerdo con los años de experiencia en el sistema educativo público. Con respecto a la formación del profesorado, los docentes asistieron a un curso de 25 horas justo antes de la implantación del programa o justo después de haber empezado el programa. Este curso fue evaluado por algunos de los profesores de mi muestra como insuficiente y poco práctico.

Una tercera limitación con respecto a la implantación del programa es consecuencia de la falta de una evaluación fiable de los conocimientos previos del alumnado antes de su entrada en el programa o al ser admitido. Sin una información detallada sobre lo que saben los alumnos, muchos profesores de «Aula de Enlace» asumen con frecuencia que los jóvenes de minorías inmigradas tienen unos conocimientos académicos por debajo de la media española (tal y como aparece en la información recogida en los cuestionarios). Sin embargo, este no es siempre el caso. Por ejemplo, muchos alumnos de origen rumano y chino de la muestra reconocieron que la educación en España es mucho más fácil que en sus países respectivos.

Por último, otro indicador que apoya el argumento de la falta de planificación y diseño tiene que ver con la evaluación del programa. Según Short y Boyson (2004: 69), «it is highly recommended that programs plan a formative evaluation process that examines student language and content development while they are in the program and after they have exited.» 
Ninguna evaluación de ese tipo se ha previsto para las «Aulas de Enlace» ni se ha incluido en la política oficial o «Instrucciones». Aunque el documento oficial tiene un apartado de evaluación, está pensada para evaluar sólo la parte administrativa del programa, es decir, su funcionamiento. No obstante, tal y como Short y Boyson (2004) han apuntado: «the formative evaluation process is an important vehicle for improving a program and verifying that it is meeting its goals and students needs, a reason why a formative evaluation of the program's implementation and the students' progress should be conducted each year.»

\section{Conclusiones}

La primera pregunta que surge del análisis de datos es si un programa que se muestra útil para tan solo un $50 \%$ de la población a la que trata de servir se puede considerar realmente efectivo. Además, este porcentaje pertenece sobre todo a alumnos de origen rumano, muchos de los cuales se habrían incorporado al sistema educativo español con éxito de todos modos. Por tanto, su éxito se debe a su propio mérito, aunque también se relaciona de algún modo con los bajos estándares del sistema educativo español.

La principal crítica que puede hacérsele al programa y a las «Aulas de Enlace» es que, aunque fue implantado para dar respuesta a la diversidad en las escuelas, dicha respuesta se ha dirigido a transformar la diversidad en homogeneidad, renunciando así a sus principios básicos. A pesar de que el discurso oficial reconoce los beneficios que la diversidad lingüística y cultural aporta a la escuela, el modo en que la política educativa ha tratado el tema es, cuanto menos, contradictoria. Por otro lado, la opción de proporcionar diferentes tipos de programas educativos a estudiantes con necesidades diversas está fuera de toda cuestión, ya que se entendería como una forma de limitar la igualdad de oportunidades de los alumnos en el sistema educativo español. Dos ideas surgen de esta percepción: (1) la expresión «igualdad de oportunidades» en este contexto ha sido malinterpretada. Aunque con el fin de evitar desigualdades en la educación todos los alumnos deberían tener acceso a las mismas oportunidades para tener éxito, esto no significa que todos hayan de seguir el mismo camino para alcanzar unos mismos objetivos; (2) la segunda idea que surge es la preocupación de aquellos que controlan la política educativa acerca del riesgo de segregación, con los consiguientes problemas sociales que podría acarrear.

Desde mi punto de vista, esta preocupación de la consejería de educación de la CAM por proporcionar igualdad de oportunidades a todos los alumnos se basa en razones políticas más que sociales. Puesto que la educación se ha convertido en un foco de conflicto entre el partido en el gobierno y la oposición, el gobierno regional debe manejar la educación de inmigrantes con cuidado, ya que podría ser acusado de segregación. Sin embargo, los alumnos inmigrantes en el programa son segregados de facto cuando se les incorpora rápidamente al aula ordinaria y se les enseña exclusivamente en español. Aunque aparentemente integrados en su clase, no tienen acceso a las ideas que allí se presentan ni herramientas suficientes para interactuar en español.

Una de las conclusiones de este estudio es que el diseño y la implantación de las «Aulas de Enlace» fue consecuencia de una serie de decisiones tomadas desde los despachos y no informada por la literatura académica. El desconocimiento de esta literatura ha dado como resultado la concepción de falsas ideas sobre el proceso de aprendizaje de una lengua, ideas que a su vez han guiado de manera errónea a los administradores encargados de hacer la política 
educativa y tomar decisiones al respecto. En primer lugar, el programa «Aulas de Enlace» es demasiado corto para producir adquisición. Una de las conclusiones más sólidas del estudio de Hakuta et al. (2000) sobre el tiempo que lleva el aprendizaje del inglés por parte de los alumnos inmigrados en Estados Unidos fue que las políticas educativas que tiendan al logro de un aprendizaje rápido de la lengua están abocadas al fracaso («policies that assume rapid acquisition of English are widely unrealistic.»). En segundo lugar, los alumnos inmigrantes pasan del «Aula de Enlace» al aula ordinaria en base a su competencia comunicativa eminentemente oral. Este hecho provoca dificultades para ponerse al día con el currículo del aula ordinaria, ya que los alumnos aún no han adquirido el nivel académico de lengua (CALP) requerido para seguir la clase regular con éxito. Por último, el programa no consideró el hecho de que no todos los alumnos aprenden de la misma manera y al mismo ritmo en la fase de diseño e implantación, lo que provoca gran frustración por parte de profesores y alumnos, que ven cuán difícil es alcanzar los objetivos propuestos por el programa.

Según lo dicho hasta ahora, queda patente que las «Aulas de Enlace» no pueden considerarse una innovación respecto a los programas de educación compensatoria más tradicionales.Aunque los profesores y administradores consideran las «Aulas» una mejora sustancial respecto a la educación compensatoria, y aunque este tipo de programa es el más frecuente en lugares con gran cantidad de población inmigrante, también es cierto que no es el más efectivo para conseguir el aprendizaje de la L2. Por otro lado, la implantación no siempre considera lo que es mejor para el alumno, sino lo mejor para la administración. En este sentido, el programa es administrativamente simple (no tiene currículo, no se necesitó contratar nuevo profesorado, etc.) y requería poco gasto. Así pues, y aunque las intenciones de los administradores fueron inicialmente buenas, se ha demostrado que sus objetivos no son realistas, tal y como ha mostrado el hecho de que sea útil para sólo la mitad de la muestra. Ante el riesgo de dejarnos guiar por falsas ideas sobre lo que significa aprender una lengua, Walqui (2000: 31) sugiere lo siguiente: «instead of basing our instructional programs on these misconceptions, we need to be guided by a solid understanding of the nature of second language acquisition informed by current research and theory.»

En resumen, se puede decir que la implantación de las «Aulas de Enlace» se llevó a cabo con prisa, lo que no permitió una planificación y un diseño adecuados. El hecho de que el programa fuera aprobado y hecho público por la consejería de educación en noviembre de 2002 e inmediatamente implantado en enero de 2003 apoya este argumento. Como consecuencia de la improvisación se dejaron muchos cabos sujetos al azar, tales como un criterio de evaluación claro y preciso, la selección del profesorado, guía para el seguimiento de los alumnos, etc. Además de esto, los principales participantes en el programa, es decir, alumnos y profesores, fueron ignorados en la fase de planificación, diseño e implantación, siendo el proceso básicamente guiado por las opiniones y las creencias de los administradores sobre la mejor manera de educar a los jóvenes inmigrantes.

Hubo tres factores que, en mi opinión, forzaron al gobierno regional de Madrid a implantar el programa Escuelas de Bienvenida y las «Aulas de Enlace» como su principal componente. En primer lugar, el creciente número de padres que comienza a retirar a sus hijos de las escuelas públicas para escolarizarlos en centros privados y privados concertados debido al alto número de alumnos inmigrantes que pueblan la educación pública. En segundo lugar, las quejas de los profesores del aula ordinaria ante la creciente diversidad del alumnado y la falta de recursos para hacer frente a ella de manera adecuada. Dado que los alumnos pueden 
incorporarse al aula en cualquier momento durante el año académico, los maestros y profesores se encuentran sin preparación suficiente para facilitarles los procesos de aprendizaje e integración. En tercer lugar, con las elecciones autonómicas cerca (mayo, 2003), el gobierno autonómico tenía una necesidad real de mostrar que podía dar respuesta a las preocupaciones de padres y profesores de una manera rápida y eficaz. Por lo tanto, si bien la implantación de un programa tan específico como este para alumnos de minorías inmigrantes en la CAM fue resultado de una necesidad real, es decir, el número creciente de alumnos que no hablan castellano en nuestras aulas, en mi opinión fue también una maniobra política para ganar electores.

Diversos sindicatos alzaron la voz contra la implantación del programa Escuelas de Bienvenida basándose en la falta de coordinación, información y recursos que había acompañado su rápida puesta en marcha. La respuesta del entonces vice-consejero de educación de la CAM (Juan González Blasco) a dichas críticas no se hizo esperar «este programa se ha estado planificando durante bastante tiempo, desde agosto» (refiriéndose a agosto de 2002)» (EL PAÍS, 16 de enero de 2003).

En conclusión, puede afirmarse que la rapidez con que se planificó e implantó el programa muestra falta de compromiso real con el éxito educativo del alumnado inmigrante, compromiso que para Walqui (2000: 208) constituye el pilar fundamental para el éxito de cualquier acción educativa llevada a cabo para este colectivo: «a strong commitment to immigrant students' educational success is ultimately the foundation of all successful programs and instruction.»

En la actualidad, y a pesar de que la respuesta mayoritaria del sistema educativo a la presencia de alumnado extranjero en nuestras aulas sigue siendo la implantación de programas de aprendizaje acelerado de español, cada vez está más claro que «el aprendizaje de una L2 por niños y jóvenes inmigrantes es un caso particular de aprendizaje de lenguas, tanto como por las condiciones en que se realiza como por la consideración social de los aprendices, o por el uso que se tiene que hacer de la nueva lengua.» (Villalba y Hernández, 2004: 1225). Según los autores, es un campo de estudio que no sólo incumbe a la pedagogía, sino también a la lingüística aplicada y a la didáctica del español, y proponen que se considere lo específico de la lengua que se utiliza para transmitir el currículum. En este sentido, y de acuerdo con las Propuestas de Alicante, «la redacción del Manifiesto de Santander (http://cvc.cervantes. es/debates/debates.asp?vdeb=27) supuso el punto culminante de lo que se podría considerar como la primera etapa en la enseñanza de segundas lenguas a inmigrantes.» Entre los retos más importantes de esta etapa está el de disponer de una adecuada tipología de programas que dé respuesta a las necesidades formativas de los estudiantes inmigrantes. Futuros estudios deberían dar respuesta, entre otras, a preguntas del tipo: ¿Cómo puede la educación fomentar el éxito en entornos de aprendizaje multilingües?, ¿Cuándo está preparado el alumno para dar el salto al aula ordinaria desde su «aula de enlace»?, ¿Qué prácticas educativas promueven el aprendizaje lingüístico específico que el alumno necesita para lograr sus objetivos académicos?, ¿De qué manera podría minimizarse el impacto de la distancia entre la lengua materna del alumno y la L2 en la práctica de aula? (ver Propuestas de Alicante). Por otro lado, existe una gran necesidad de estudios longitudinales que investiguen los efectos a largo plazo de los programas para alumnado extranjero inmigrado. 


\section{Referencias bibliográficas}

Cenoz, J. and F. Genesee (1998): Beyond bilingualism: multilingualism and multicultural education. Clevedon, England, Multilingual Matters.

Coelho, E. (2004): Adding English: a guide to teaching in multilingual classrooms. Toronto, Pippin Publishing Corporation.

Creswell, J.W. (1998): Qualitative inquiry and research design: choosing among five traditions. Thousand Oaks, CA, Sage.

Cummins, J. (1979a): «Linguistic interdependence and the educational development of bilingual children», Review of Educational Research, 49, págs. 222-251.

Cummins, J. (1979b): «Cognitive/academic language proficiency, linguistic interdependence, the optimum age question and some other matters», Working Papers on Bilingualism, 19, págs. 197-205.

Cummnis, J. (1980): «The construct of language proficiency in bilingual education». En Alatis, J.E. (ed.): Georgetown University Round Table on Languages and Linguistics 1980. Washington, D.C, Georgetown University Press.

Cummins, J. (1981a): Bilingualism and minority language children. Toronto, Ontario Institute for Studies in Education.

Cummnis, J. (1981b): «Age of arrival and immigrant second language learning in Canada: A reassessment», Applied Linguistics, 1, págs. 132-149.

Cummins, J. (1983): «Language proficiency and academic achievement». En Oller, J.W. (ed.): Issues in language testing research. Rowley, MA, Newbury House, págs. 108-30.

Cummins, J. (1992): «Language proficiency, bilingualism and academic achievement». En RichardAmato, P.A y M.A. Snow (eds.): The multicultural classroom: Readings for content-area teachers. New York, Longman.

Cummins, J. (2000): Language, power and pedagogy. Bilingual children in the crossfire. Clevedon, England, Multilingual Matters.

Dörnyei, Z. (2003): Questionnaires in second language research. Construction, administration, and processing. Mahwah, New Jersey, Lawrence Erlbaum Associates, Inc., Publishers.

Gardner, R.C. and W.E. Lambert (1972): Attitudes and motivation in second language learning. Rowley, Mass, Newbury House.

Genesee, F. (ed.) (1994): Educating second language children. The whole child, the whole curriculum, the whole community. New York, Cambridge University Press.

Genesee, F. (1999): Program alternatives for linguistically diverse students. Santa Cruz, CA, Center for Research on Education, Diversity and Excellence.

Guba, E.G. y Y.S. Lincoln (1989): Fourth generation evaluation. Thousand Oaks, CA, Sage.

Guba, E.G. y Y.S. Lincoln (1994): «Competing paradigms in qualitative research». En Denzin, N.K. y Y.S. Lincoln (eds.): Handbook of qualitative research (2nd edition). Thousand Oaks, CA, Sage.

Hakuta, K., Y.G. Butler y D. Witt (2000): How long does it take English learners to attain proficiency? Santa Barbara, CA, University of California Linguistic Minority Research Institute: http://faculty. ucmerced.edu/khakuta/research/publications.html (02-10-2009).

Hall, J.K. y L.S. Verplaetse (eds.) (2000): Second and foreign language learning through classroom interaction. Mahwah, NJ, Lawrence Erlbaum.

Hatch, E. (1992): Discourse and language education. Cambridge, Cambridge University Press.

Johnstone, B. (2000): Qualitative methods in sociolinguistics. Oxford, Oxford University Press.

King, J.A., L.L. Morris y C.T. Fitz-Gibbon (1987): How to assess program implementation. Thousand Oaks, CA, Sage.

Lambert, W.E. y R. Tucker (1972): Bilingual education of children. The St. Lambert experiment. Rowley, MA, Newbury House. 
Larsen-Freeman, D. (2000): Techniques and principles in language teaching. Oxford, Oxford University Press.

Lee, J.F. y B. VanPatten (1995): Making communicative language teaching happen. New York, McGraw-Hill.

Lenneberg, E. (1967): The biological foundations of language. New York, John Wiley.

Lightbown, P.M. y N. Spada (1999): How languages are learned (2nd edition). Oxford, Oxford University Press.

Long, M.H. (1983): «Does second language instruction make a difference? A review of the research». TESOL Quarterly, 17, págs. 359-82.

Long, M.H. (1990): «Maturational constraints on language development. Studies in Second Language Acquisition, 12 (3), págs. 251-85.

Lora-Tamayo D’Ocón, G. (2003a): «Evolución reciente y perfil de la población extranjera en Madrid». Migraciones, 13, págs. 7-59.

Merriam, S.B. (1988): Case study research in education: A qualitative approach. San Francisco, Jossey-Bass.

Nieto, S. (1994): «Lessons from students on creating a chance to dream», Harvard Educational Review, vol. 64, 4, Winter 1994, págs. 392-426.

Odlin, T. (1989): Language transfer. Cambridge, Cambridge University Press.

Oxford, R. y J. Shearin (1994): «Language learning motivation: Expanding the theoretical framework», Modern Language Journal, 78 (1), págs. 12-28.

Patton, M. Q. (2002): Qualitative research and evaluation methods (3rd edition). Thousand Oaks, Sage.

Pica, T. (1994): «Research on negotiation. What does it reveal about second language acquisition?», Language Learning, 44, págs. 493-527.

Plaut, S. y N.S. Sharkey (eds.) (2003): «Education policy and practice. Bridging the divide», Harvard Education Review, Reprint Series, $\mathrm{n}^{\circ} 37$.

Ramírez, J.D. (1992): «Executive summary», Bilingual Research Journal, 16 (1 y 2), págs. 1-62.

Ruiz, R. (1984): «Orientations in language planning», NABE Journal, 8, págs. 15-34.

Short, D.J. (2002): «Newcomer programs: An educational alternative for secondary immigrant students», Education and Urban Society, 34 (2), págs. 173-198.

Short, D.J. y B.A. Boyson (2004): Creating access. Language and academic programs for secondary school newcomers. Washington, DC, Center for Applied Linguistics.

Shrum, J.L. y E.W. Glisan (2000): Teacher's handbook: Contextualized language instruction (2nd edition). Boston, MA, Heinle \& Heinle.

Soriano Ayala, E. (2000): «Multicultural education in primary schools in Almería, Spain». En Trueba, E.T y L.I. Bartolomé: Immigrant Voices: In Search of Educational Equity. Oxford, Rowman \& Littlefield Publishers.

Swain, M. y S. Lapkin (1991): «Heritage Language Children in an English-French bilingual program», The Canadian Modern Languages Review: 47 (4), págs. 635-641.

Thomas, W.P. y V. Collier (1997): School effectiveness for language minority students. (NCBE Resource Collection Series No. 9). Washington, DC, National Clearinghouse for Bilingual Education.

Thomas, W.P. y V. Collier (2002): A national study of school effectiveness for language minority students'long-term academic achievement. Santa Cruz, CA, Center for Research on Education, Diversity and Excellence: http://www.crede.ucsc.edu/research/llaa/1.1_final.html (02-10-2009).

Tucker, R.G. y A. D'Anglejan (1972): «An approach to bilingual education». En M. Swain (ed.): Bilingual schooling: some experiences in Canada and the United States. Ontario: Ontario Institute for Studies in Education Symposium Series/1.

Valladares, I. (2005): «Inmigración y escuela. Cuestiones básicas y algunas ideas», Tintero. Revista Digital, 56, págs. 23-25. 
Van Manen, M. (1990): Researching lived experience: human science for an action sensitive pedagogy. New York, State University of New York.

Villalba, F. y M $\mathrm{M}^{\mathrm{a}}$ T. Hernández (2004): «La enseñanza del español a inmigrantes en contextos escolares». En Sánchez Lobato, J. e I. Santos Gargallo (dirs): Vademécum para la formación de profesores. Enseñar español como segunda lengua (L2)/lengua extranjera (LE). Madrid, SGEL, págs. $1225-1258$.

VV.AA. (2004): Manifiesto de Santander: http://cvc.cervantes.es/obref/inmigracion/documentos/manifiesto.htm (08-10-2009)

VV.AA. (2006): Propuestas de Alicante: http://www.ua.es/dfelg/lengua/EncuentroL2/PROPUESTAS. ALICANTE.pdf (08-10-2009)

Vygotsky, L.S. (1978): Mind in society. Cambridge, MA, Harvard University Press.

Walqui, A. (2000): Access and engagement. Program design and instructional approaches for immigrant students in secondary school. Washington, DC, Center for Applied Linguistics.

Wong Fillmore, L. (1991): «Second language learning in children: A model of language learning in social context». En Bialystok, E. (ed.): Language processing in bilingual children. Cambridge, Cambridge University Press. 